\title{
Prevalence of Nocturia in a Korean Population Aged 40 to 89 Years
}

\author{
Myung-Soo Choo, ${ }^{1}$ Ja Hyeon $\mathrm{Ku},{ }^{2}$ Choal Hee Park, ${ }^{3}$ You Sik Lee, ${ }^{4}$ Kyu-Sung Lee, ${ }^{4}$ \\ Jeong Gu Lee, ${ }^{5}$ and Won-Hee Park ${ }^{6 *}$ \\ ${ }^{1}$ Department of Urology, University of Ulsan College of Medicine, Seoul, Korea \\ ${ }^{2}$ Department of Urology, Seoul National University College of Medicine, Seoul, Korea \\ ${ }^{3}$ Department of Urology, Keimyung University School of Medicine, Daegu, Korea \\ ${ }^{4}$ Department of Urology, SungKyunKwan University School of Medicine, Seoul, Korea \\ ${ }^{5}$ Department of Urology, College of Medicine Korea University, Seoul, Korea \\ ${ }^{6}$ Department of Urology, Inha University College of Medicine, Incheon, Korea
}

\begin{abstract}
Aims: The purpose of this study was to evaluate the prevalence of nocturia in Korea, to examine the relationship between nocturia and demographic variables, and to determine the impact of nocturia on daily living. Methods: A national telephone survey using quota sampling methods was conducted in Korea. The clinically validated computer-assisted telephone interview approach was used for the survey. Results: Of 2005 subjects (1,005 women and 1,000 men) interviewed, 33.5\% reported voiding once per night and $48.2 \%$ twice or more per night. Nocturia increased with age among both genders and was more common among young women than young men. In all subjects, multivariate analysis indicated that female gender, older age and an overweight condition were independent risk factors. Body mass index was associated with an increased likelihood of nocturia in male but not in female subjects. In female subjects, the likelihood of at least one night-time void was related to delivery number (odds ratio 1.17, 95\% confidence interval 1.04-1.32). An impact of nocturia on daily life was reported by $14.6 \%$ of subjects and only $3.8 \%$ (4.0\% of men and $3.7 \%$ of women) sought medical care. Commonly reported reasons for not seeking medical care were the belief that nocturia is a normal consequence of aging or is not a disease (92.8\% of subjects reporting an impact of nocturia on daily life). Conclusions: Although nocturia is highly prevalent in the Korean population, it has only a minor impact on daily living, and few individuals seek medical care. Our study provides a valuable insight into the need for tailored nocturia education addressed to the population who view the condition as trivial. Neurourol. Urodynam. 27:60-64, 2008. (c) 2007 Wiley-Liss, Inc.
\end{abstract}

Key words: nocturia; quality of life; prevalence; epidemiology; survey

\section{INTRODUCTION}

Nocturia is defined as waking at night to void urine. ${ }^{1}$ It has been suggested that nocturia is one of the most bothersome symptoms of lower urinary tract symptoms in men and women. $^{2}$ Rising at night to void urine increases the risk of incurring personal injuries, particularly important in elderly subjects, in whom the condition is associated with a greater risk of falls ${ }^{3}$ and hip fractures. ${ }^{4}$ Nocturia also has other profound implications for sufferers, exerting detrimental effects on quality of life and sleep patterns, and is associated with increased mortality. ${ }^{5}$

However, not all patients with nocturia are bothered by their condition and the degree to which they are bothered is likely to be associated with the degree of nocturia. In addition, several surveys have shown that racial differences exist both in nocturia risk factors and the prevalence of nocturia. These racial differences reflect cultural differences in the perception of nocturia and its importance. ${ }^{6-8}$

To date, although epidemiologic studies of lower urinary tract symptoms (LUTS) have been performed in many countries, targeted symptoms and populations were generally limited. In addition, the few studies on female nocturia suggest that male and female nocturia may not have identical etiologies. ${ }^{9}$ Furthermore, there is a lack of epidemiological data in a Korean population. The purpose of this study was to evaluate the prevalence of nocturia in an adult Korean national community sample. In addition, the relationship between nocturia and demographic variables including gender, age and body mass index was examined. Lastly, we determined the impact of nocturia on daily living and willingness to seek medical consultation.

\section{MATERIALS AND METHODS}

To assess the prevalence of nocturia, a national telephone survey using quota sampling methods was conducted in 2000 A random sample of adults aged 40-89 years was generated from the target population using a stratified approach based on geographical, gender, and age variables. The study included all territories of South Korea. Within each territory and gender, respondents were stratified into five age groups: $40-49$ years, 50-59 years, 60-69 years, 70-79 years and 80-89 years. Prevalence estimates were weighted for the probability of selection on the basis of household size and for the age and sex structure of the resident population of Korea, on the basis of 1995 Census data. Then, telephone numbers were chosen

Rodney Appell led the review process.

This study was supported by the Korean Continence Society and Ferring Pharmaceuticals (Korea grant).

Abbreviations: LUTS, lower urinary tract symptoms; OR, odds ratio; CI, confidence interval; SD, standard deviation; ICS, International Continence Society. ${ }^{*}$ Correspondence to: Won-Hee Park, MD, Department of Urology, Inha University Hospital, 7-206, 3-ga, Sinheung-dong, Jung-gu, Incheon 400-103, Korea.

E-mail: drwonhee@inha.ac.kr

Received 24 November 2006; Accepted 16 April 2007 Published online 12 June 2007 in Wiley InterScience (www.interscience.wiley.com)

DOI 10.1002/nau.20458 
randomly using a computer program based on the telephone number listings of Korea in 2000. A clinically validated computer-assisted telephone interview approach was used in the survey. ${ }^{10-13}$

Trained interviewers called households between 18.00 and 22.00 during weekdays and weekends of the study period, to avoid over-representation of housewives. In this survey, 14,559 telephone calls were made and the respondents stratified by their age group. A total of 2,005 adults (1,005 women and 1,000 men) among 14,559 participants 40 years or older completed the telephone interview. The study participants were randomly selected with sampling errors within $2.2 \%$. Interviews generally lasted between 30 and $60 \mathrm{~min}$. An unanswered call was re-attempted at least three times before the telephone number was defined as invalid. Data were collected from only one respondent in each household.

The questionnaire was carefully developed by the authors and evaluated for reliability and validity in advance of the survey, through pilot studies using symptomatic and asymptomatic nonmedical personnel. Data were collected using a structured interview schedule and comprised information on demography and nocturia in the last four weeks. For respondents answering "yes" when asked if they experienced nocturia, a series of additional questions was asked to obtain detailed information on nocturia. Nocturia was identified from the question: "how many times did you most typically get up to urinate from the time you went to bed at night until the time you got up in the morning?" Then, respondents were asked questions about the number of nocturia episodes. Additional questions were asked to determine reasons for waking at night, the impact of nocturia, and willingness to seek medical consultation.

The prevalence of nocturia and its impact were summarized using descriptive statistics. Descriptive statistics were completed using SPSS 11.0 (SPSS, Inc., Chicago, IL, USA) and are reported as percentages. Subjects were stratified by age into five groups: $40-49,50-59,60-69,70-79$ and $80-89$ years of age. Data for nocturia were extracted and comparisons of the prevalence of nocturia in both sexes in different age decades were analyzed using the Armitage test. To determine the factors that were associated with the number of night-time voids, we estimated odds ratios (ORs) and $P$ values for trends using multivariate logistic regression analysis. Subject demographics (gender, age, body mass index) were individually entered into the model. Body mass index was classified as less than or greater than $25.0 \mathrm{~kg} / \mathrm{m}^{2}$. For women only, delivery number was used as a component of demographics in the multivariate model. The associations between subject demographics and the numbers of night-time voids were determined using maximum likelihood estimates of relative risk with 95\% confidence intervals (CIs), on the basis of the logistic regression model. The CIs were predicated from the standard error of measurements of the coefficients and a normal approximation. A 5\% level of significance was used throughout, and all statistical tests were two-sided.

\section{RESULTS}

The mean and median respondent ages were 59.7 years (standard deviation [SD] 11.5) and 60.0 years for men, respectively, and 59.4 years (SD 11.6) and 59.0 years for women, respectively. The prevalence of nocturia in each age cohort, and the frequency of nocturia, are presented in Figure 1. Of the respondents, $27.3 \%$ did not report any nocturia, 33.5\% reported voiding once per night and $48.2 \%$ twice or more per night (Fig. 1A). Nocturia increased with age among both genders $(P<0.001)$. Nocturia was more common among women than
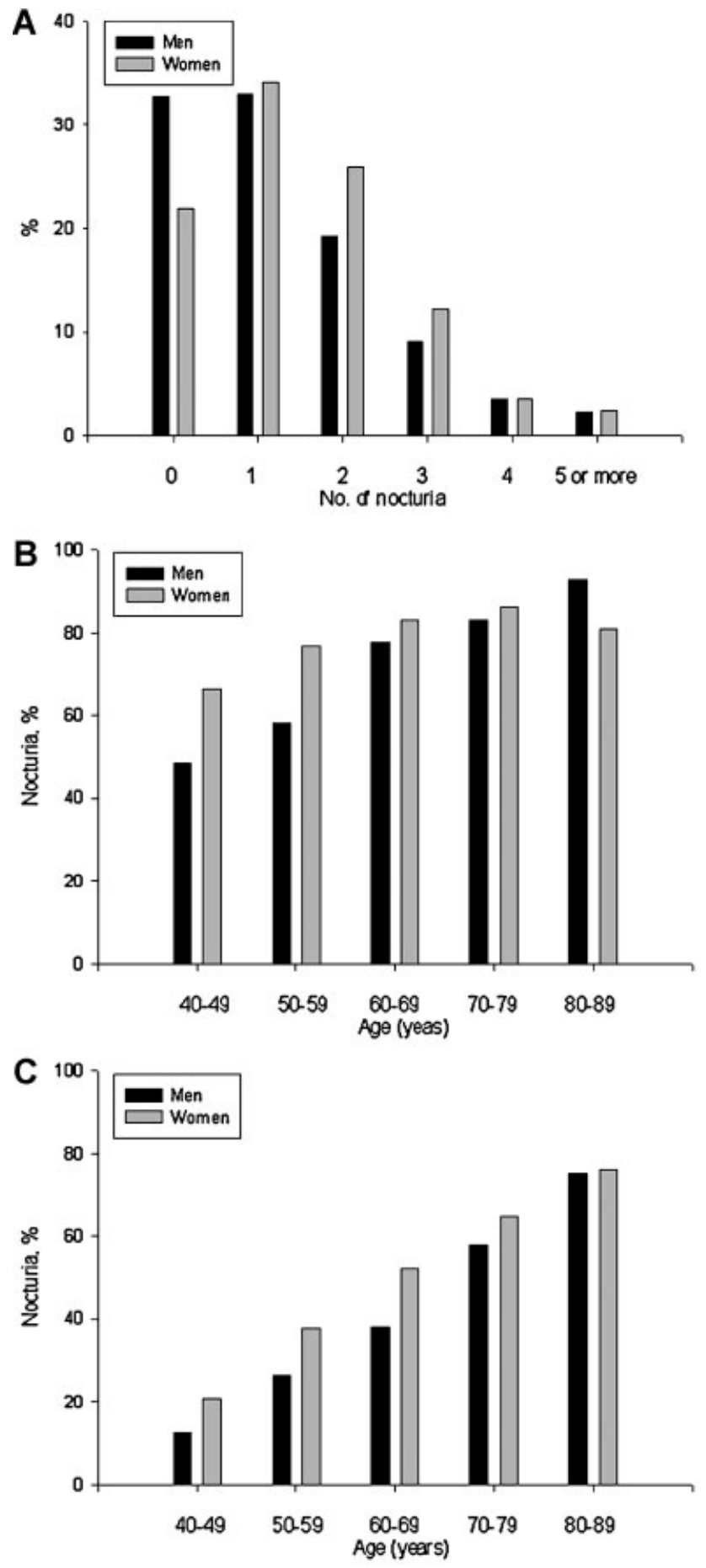

Fig. 1. A. Distribution of severity of nocturia. B. Overall prevalence of nocturia (defined as at least 1 void per night). C. Overall prevalence of nocturia (as at least 2 voids per night).

among men in the two younger age categories $(P<0.001$ for subjects 40 to 49 years of age and $P<0.001$ for people 50 to 59 years of age). The prevalence in men began to approach that in women in the age group 60 to 69 years and reached the same prevalence at age 70 to 79 years (Fig. 1B). When nocturia was defined as at least two voids per night, nocturia increased linearly with age among both genders $(P<0.001)$. Nocturia twice per night was more common among young women than 
TABLE I. Multivariate Logistic Regression Analysis of the Influence on at Least One or $\geq$ Two Night-Time Voids

\begin{tabular}{|c|c|c|c|c|c|c|}
\hline & \multicolumn{2}{|l|}{ Total } & \multicolumn{2}{|l|}{ Men } & \multicolumn{2}{|l|}{ Women } \\
\hline & Adjusted OR (95\% CI) & $P$-value & Adjusted OR (95\% CI) & $P$-value & Adjusted OR (95\% CI) & $P$-value \\
\hline \multicolumn{7}{|c|}{ At least one void/night } \\
\hline \multicolumn{7}{|l|}{ Sex } \\
\hline Male & 1.000 & & & & & \\
\hline Female & $1.796(1.457-2.213)$ & $<0.001$ & & & & \\
\hline \multicolumn{7}{|l|}{ Age (years) } \\
\hline $40-49$ & 1.000 & & 1.000 & & 1.000 & \\
\hline $50-59$ & $1.536(1.181-1.998)$ & 0.001 & $1.491(1.044-2.130)$ & 0.028 & $1.495(0.990-2.256)$ & 0.056 \\
\hline $60-69$ & $3.133(2.347-4.182)$ & $<0.001$ & $3.829(2.589-5.663)$ & $<0.001$ & $1.853(1.149-2.990)$ & 0.011 \\
\hline $70-79$ & $4.381(3.180-6.036)$ & $<0.001$ & $5.285(3.431-8.139)$ & $<0.001$ & $2.392(1.371-4.171)$ & 0.002 \\
\hline $80-89$ & $5.222(2.160-12.624)$ & $<0.001$ & $12.536(2.922-53.784)$ & $<0.001$ & $1.076(0.316-3.666)$ & 0.907 \\
\hline \multicolumn{7}{|l|}{ BMI $\left(\mathrm{kg} / \mathrm{m}^{2}\right)$} \\
\hline$<25$ & 1.000 & & 1.000 & & 1.000 & \\
\hline$\geq 25$ & $1.506(1.165-1.947)$ & 0.002 & $1.503(1.059-2.132)$ & 0.023 & $1.463(0.996-2.150)$ & 0.053 \\
\hline Delivery no. & & & & & $1.170(1.038-1.318)$ & 0.010 \\
\hline \multicolumn{7}{|c|}{ At least twice/night } \\
\hline \multicolumn{7}{|c|}{ Sex } \\
\hline Male & 1.000 & & & & & \\
\hline Female & $1.591(1.307-1.935)$ & $<0.001$ & & & & \\
\hline \multicolumn{7}{|l|}{ Age (years) } \\
\hline $40-49$ & 1.000 & & 1.000 & & 1.000 & \\
\hline $50-59$ & $2.356(1.738-3.195)$ & $<0.001$ & $2.509(1.565-4.023)$ & $<0.001$ & $2.337(1.557-3.507)$ & $<0.001$ \\
\hline $60-69$ & $4.146(3.079-5.584)$ & $<0.001$ & $4.430(2.807-6.991)$ & $<0.001$ & $3.937(2.624-5.907)$ & $<0.001$ \\
\hline $70-79$ & $8.343(6.131-11.353)$ & $<0.001$ & $9.805(6.161-15.603)$ & $<0.001$ & 7.448 (4.849-11.439) & $<0.001$ \\
\hline $80-89$ & $15.012(7.406-30.433)$ & $<0.001$ & $19.044(7.383-49.121)$ & $<0.001$ & $10.741(3.638-31.716)$ & $<0.001$ \\
\hline \multicolumn{7}{|l|}{ BMI $\left(\mathrm{kg} / \mathrm{m}^{2}\right)$} \\
\hline$<25$ & 1.000 & & 1.000 & & 1.000 & \\
\hline$\geq 25$ & $1.391(1.105-1.750)$ & 0.005 & $1.495(1.064-2.099)$ & 0.020 & $1.334(0.973-1.828)$ & 0.083 \\
\hline Delivery no. & & & & & $0.990(0.965-1.015)$ & 0.431 \\
\hline
\end{tabular}

OR, odds ratio; CI, confidence interval; BMI, body mass index.

among young men $(P=0.012$ for women 40 to 49 years, $P=0.006$ for women 50 to 59 years and $P=0.002$ for women 60 to 69 years). Men reported the same frequency of nocturia as did women when both genders were in their $70 \mathrm{~s}$ to $80 \mathrm{~s}$ (Fig. 1C).

In all subjects, multivariate analysis indicated that gender, age and body mass index were independent risk factors. In male subjects only, age and body mass index were also associated with an increased likelihood of at least one nighttime void. However, in female subjects only, body mass index had no statistical significance. In these subjects, the likelihood of at least one night-time void was related to delivery number (OR 1.17, 95\% CI 1.04 to 1.32). When two or more night-time voids was considered to represent nocturia, age only was associated with risk of nocturia in female subjects (Table I).

Nocturia was stated by most respondents as a reason for waking at night, but there were some other reasons for waking, including insomnia. An impact on daily life by nocturia was reported in $14.6 \%$ of subjects. When questions regarding medical treatment were asked, only $3.8 \%$ (4.0\% of men and $3.7 \%$ of women) reported that they had sought medical care. More subjects (7.2\%) with at least three nighttime voids reported that they would seek help from a health professional $(P<0.001)$. Commonly reported reasons for not seeking medical care were the belief that nocturia is a normal consequence of aging or is not a disease (92.8\%) (Table II).

\section{DISCUSSION}

Epidemiologic surveys are important for identifying the characteristics of diseases and their possible causes, and for estimating social impacts of diseases. The estimated prevalence of nocturia has varied, largely due to different definitions of the condition. The standard definition for nocturia, introduced only recently by the International Continence Society (ICS), is waking at night to void, and applies to any number of voids at any time during a night's sleep; each void is preceded by and followed by sleep and the person is awake before voiding. ${ }^{1}$ However, in most earlier studies, nocturia was defined as night-time voiding at least twice per night. Importantly, estimates of the prevalence of nocturia varied depending on the definition of nocturia and age group surveyed. Thus, in the present study, we used this definition (night-time voiding at least twice per night) in the analysis although we also used the ICS definition of nocturia.

In our cohort, the prevalence of nocturia increased with subject age. It has been consistently reported that the incidence of nocturia and the total number of voiding episodes increases with age. ${ }^{14-17}$ Recently, Hakkinen et al. ${ }^{18}$ reported that while mild nocturia is common in young, mature Finnish male individuals (37\% of 50-year-old men) and increases sharply during the decade from 50 to 60 years (to $60 \%$ in the same men at age 60), elderly men experience the greatest increase in severe (three or four episodes nightly) nocturia between ages 70 to 80 years (7\% at 70 years; $19 \%$ at 80 years). As results of an age-associated diminution of renal concentrating capacity, as well as diminished sodium-conserving ability, loss of the circadian rhythm of antidiuretic hormone secretion, attenuated secretion of renin-angiotensin-aldosterone, and increased secretions of atrial natriuretic hormone, researchers have previously noted an age-associated alteration in the circadian rhythm of water excretion, resulting in increased nighttime urine production in older people. ${ }^{19}$ Therefore, age-related physiological changes can alter the regular pattern of urine excretion and lead to an increased nocturnal frequency of voiding. Additionally, aging is associated with anatomical and physiological changes of 
TABLE II. Reasons, Impact, and Willingness to Seek Medical Consultation

\begin{tabular}{|c|c|c|c|c|}
\hline & \multicolumn{3}{|c|}{ No. of night-time voids } & \multirow[b]{2}{*}{$P$-value* } \\
\hline & One $(n=672)$ & Two $(n=453)$ & Three $(\mathrm{n}=332)$ & \\
\hline Reasons for waking at night & & & & 0.345 \\
\hline To void & $517(76.9 \%)$ & $334(73.7 \%)$ & $252(75.9 \%)$ & \\
\hline Habitually & $94(14.0 \%)$ & 77 (17.0\%) & 46 (13.9\%) & \\
\hline Insomnia & $60(8.9 \%)$ & $37(8.2 \%)$ & $29(8.7 \%)$ & \\
\hline Have worries about wet due to incontinence & $1(0.1 \%)$ & $5(1.1 \%)$ & $5(1.5 \%)$ & \\
\hline Others & - & - & - & \\
\hline Impact on daily living & & & & $<0.001$ \\
\hline None & $629(93.6 \%)$ & $401(88.5 \%)$ & $214(64.5 \%)$ & \\
\hline Only a little & $38(5.7 \%)$ & $46(10.2 \%)$ & $82(24.7 \%)$ & \\
\hline Some & $4(0.6 \%)$ & - & $25(7.5 \%)$ & \\
\hline A lot & $1(0.1 \%)$ & $6(1.3 \%)$ & $11(3.3 \%)$ & \\
\hline Seeking medical consultation & & & & $<0.001$ \\
\hline Have no willingness & $647(96.2 \%)$ & $421(92.9 \%)$ & $278(83.7 \%)$ & \\
\hline Have willingness & $13(1.9 \%)$ & $18(4.0 \%)$ & $24(7.2 \%)$ & \\
\hline Already had consulted & $12(1.8 \%)$ & $14(3.1 \%)$ & $30(9.0 \%)$ & \\
\hline
\end{tabular}

*Armitage test.

Data presented are numbers (\%).

the urinary tract, which predispose towards increased urinary frequency without affecting urine volume. The total urine output over $24 \mathrm{~h}$ does not change with increasing age but the diurnal rhythm of urine output becomes altered in many elderly patients. ${ }^{20,21}$

Contrary to our results, nocturia has been reported with similar frequency among men and women in several studies. $^{22-25}$ In a Japanese study, Yoshimura et al. ${ }^{25}$ did not observe gender differences in nocturia prevalence based on a health screening questionnaire. They reported nocturia (at least two voids per night) in $28.5 \%$ of respondents in a study of 4,568 men and 1,949 women.Schatzl et al. ${ }^{23}$ reported that $11 \%$ of men and $12 \%$ of women in a general population from the Vienna area had nocturia, defined as at least two voids per night. Coyne and colleagues reported similar results, with nocturia affecting men and women equally. ${ }^{24}$ However, Van Dijk et al. ${ }^{26}$ also observed that in the Dutch adult population nocturia was more common among women than among men, with $9 \%$ of men and $16 \%$ of women reporting nocturia. Furthermore, in Sweden, Rembratt et al. ${ }^{27}$ found that men had more nocturia than had women (31\% vs. $26 \%$, at least two voids per night) in 2,866 subjects older than 65 years. Bing et al. ${ }^{28}$ also reported that nocturia was slightly more severe in men than in women, although there was no difference between genders in overall prevalence, in a Danish population of men and women aged 60-80 years. In a study conducted in Finland, nocturia was more common among young women than young men, but the prevalence among men became comparable to that of women as men reached middle age, and exceeded that of women in men aged over $60 .^{29}$

Similarly, we also found that nocturia was more common among young women than young men, but we found no gender differences in the older population. Mueller et al. ${ }^{30}$ previously reported urinary habits of asymptomatic adults. Men had higher total fluid intake and mean voided volume than did women. Women voided more frequently than did men and had more voids per liter of fluid intake. Latini et al. ${ }^{31}$ found that urinary frequency was related to fluid intake in asymptomatic men. These data suggested significant gender effects on urinary habits, with females tending to void more often and at lower mean volumes.

In the present study, multivariate analysis indicated that body mass index was an independent risk factor in all subjects. Those overweight or obese $\left(\geq 25 \mathrm{~kg} / \mathrm{m}^{2}\right)$ were at 1.5 -fold risk (OR 1.51, 95\% CI 1.17 to 1.95 ) of at least one nighttime void and at 1.4-fold risk (OR 1.39, 95\% CI 1.11-1.75) of two or more night-time voids, compared with subjects of normal weight. However, while body mass index was associated with an increased likelihood of at least one nighttime void in male subjects, it had no statistical significance in female subjects. Prostate size or prostate growth rate has been found to be positively associated with anthropometric measures such as body mass index or waist-to-hip ratio. ${ }^{32}$ Obesity in adult males may thus be associated with a higher prevalence of nocturia, and an association between nocturia and body mass index might be expected in adult men. However, Asplund and Aberg ${ }^{33}$ demonstrated that nocturnal micturition is increased by raised body mass index in women. Furthermore, Tikkinen et al. $^{34}$ suggested that obesity was associated with increased nocturia more strongly among women than among men. Existing data on nocturia are difficult to interpret, but a number of possible explanations for our results may be proposed. The diverse findings might partly be explained by different research designs and populations. It is also possible that our findings reflect a true difference in the cause of nocturia between countries, because it is currently unknown whether any differences in nocturia etiology exist between countries, races, or social groups. Since the current ICS definition of nocturia does not include causality, future trials should include efforts to identify risk factors for nocturia. Finally, we found that the likelihood of at least one night-time void was related to delivery number in female subjects. To our knowledge, this finding has not been described previously. More studies are needed on the relationship between delivery number and nocturia.

The present study provides some data on reasons for waking at night. It is clear that most (75\%) participants with nocturia woke because of an urge to void while $25 \%$ woke at night for other reasons. In this study, nocturia of one or two voids/night was not bothersome in approximately $90 \%$ of subjects, although the impact on daily living and willingness to seek medical consultation increased with the severity of nocturia. When considering nocturia in relation to other urinary symptoms in men with benign prostatic hyperplasia, Eckhardt et al. ${ }^{35}$ noted nocturia to be the second most bothersome symptom after urinary urgency. Several community surveys found that two or more voids/night were 
"troublesome".,36 While the prevalence of nocturia was slightly higher in the Netherlands than in other countries, Dutch people, however, felt less bothered by having to void at night. ${ }^{26}$ The distinct Korean cultural background may affect our results, as may concerns with diseases associated with nocturia, or perceptions of aging, because many of our subjects reported that their symptoms were not bothersome enough to seek medical care. However, a lack of awareness or misconceptions about nocturia might also influence our findings because the vast majority of our subjects believed that nocturia was a normal consequence of aging or was not a disease. Bing et al. ${ }^{28}$ also found that acceptance of the symptom as part of aging might explain why relatively few study participants with nocturia had consulted a physician. Another possibility was a lack of knowledge of treatment opportunities. Similarly, a recent study by Chen et al. ${ }^{37}$ revealed that in women living in Taiwan, access to medical information was associated with medical consultation, while the lack of knowledge that nocturia was treatable appeared to be an important barrier to medical consultation.

This study is the first to examine the prevalence of nocturia in Korean adults. The study design has limitations, however. First, our study did not use a validated questionnaire because no disease-specific questionnaire has been psychometrically validated in the Korean language. Second, a particular weakness is the low level of interview responses. The statistical consequence of increased sampling error is that it makes it more difficult to define small but real differences as significant. A more serious consequence of non-response is a non-response bias. Non-response bias occurs if the subjects responding to a survey are consistently different from those who do not respond. We suspect that our non-responders were not interested in nocturia and might not be eager to talk about the condition. Unfortunately, the importance of this effect in our survey is unclear because we have no demographic information on the nonrespondents which might allow a comparison with respondents. Finally, in the present study, other factors that can fragment sleep, and that may be associated with nocturia, including thirst, anxiety, worry, obstructive sleep apnea, cardiovascular disease, peripheral edema, leg tingling or muscle cramps were not evaluated. ${ }^{28,38,39}$ Nocturia is not merely a function of aging and generally occurs for a combination of reasons, many of which are amenable to some sort of intervention. Answers to the questions posed above will allow better evaluation of potential therapies and more effective management of this condition.

\section{CONCLUSIONS}

The present study has provided insights into the prevalence and details of nocturia in the Korean population. Although nocturia is highly prevalent in Korea, it has only minor impact on daily living, and few individuals seek medical care. Our study provides a valuable insight into the need for tailored nocturia education of this population.

\section{REFERENCES}

1. van Kerrebroeck P, Abrams P, Chaikin D, et al. The standardisation of terminology in nocturia: report from the Standardisation Sub-committee of the International Continence Society. Neurourol Urodyn 2002;21:179-83.

2. Johansson C, Hellstrom L, Ekelund P, et al. Urinary incontinence: a minor risk factor for hip fractures in elderly women. Maturitas 1996;25:21-8.

3. Stewart RB, Moore MT, May FE, et al. Nocturia: a risk factor for falls in the elderly. J Am Geriatr Soc 1992;40:1217-20

4. Weiss JP, Blaivas JG. Nocturia. J Urol 2000;163:5-12.

5. Asplund R. Mortality in the elderly in relation to nocturnal micturition. BJU Int 1999:84:297-301.

6. Mariappan P, Chong WL. Prevalence and correlations of lower urinary tract symptoms, erectile dysfunction and incontinence in men from a multiethnic
Asian population: Results of a regional population-based survey and comparison with industrialized nations. BJU Int 2006;98:1264-8.

7. Liew LC, Tiong HY, Wong ML, et al. A population study of nocturia in Singapore. BJU Int 2006;97:109-12.

8. Kawauchi A, Tanaka Y, Soh J, et al. Causes of nocturnal urinary frequency and reasons for its increase with age in healthy older men. J Urol 2000;163. $81-4$.

9. Hvistendahl GM, Djurhuus JC. Female nocturia. Int Urol Nephrol 2002;33: 179-86.

10. Duffy JC, Waterton JJ. Under-reporting of alcohol consumption in sample surveys: the effect of computer interviewing in fieldwork. Br J Addict 1984 79:303-8.

11. Turner $\mathrm{CF}, \mathrm{Ku} \mathrm{K}$, Rogers $\mathrm{SM}$, et al. Adolescent sexual behavior, drug use, and violence: increased reporting with computer survey technology. Science 1998;280:867-73.

12. Des Jarlais DC, Paone D, Milliken J, et al. Audio-computer interviewing to measure risk behaviour for HIV among injecting drug users: a quasirandomised trial. Lancet 1999;353:1657-61.

13. Kissinger P, Rice J, Farley T, et al. Application of computer-assisted interviews to sexual behavior research. Am J Epidemiol 1999;149:950-4.

14. Britton JP, Dowell AC, Whelan P. Prevalence of urinary symptoms in men aged over 60. Br J Urol 1990;66:175-6.

15. Homma Y, Imajo C, Takahashi S, et al. Urinary symptoms and urodynamics in a normal elderly population. Scand J Urol Nephrol Suppl 1994;157:27-30.

16. Sagnier PP, MacFarlane G, Richard F, et al. Results of an epidemiological survey using a modified American Urological Association symptom index for benign prostatic hyperplasia in France. J Urol 1994;151:1266-70.

17. Malmsten UGH, Milsom I, Molander U, et al. Urinary incontinence and lower urinary tract symptoms: an epidemiological study of men aged 45 to 99 years. J Urol 1997:158:1733-7.

18. Hakkinen JT, Hakama M, Shiri R, et al. Incidence of nocturia in 50 to 80 -yearold Finnish men. J Urol 2006;176:2541-5.

19. Miller M. Nocturnal polyuria in older people: pathophysiology and clinical implications. J Am Geriatr Soc 2000;48:1321-9.

20. Larsson G, Victor A. Micturition patterns in a healthy female population, studied with a frequency/volume chart. Scand J Urol Nephrol Suppl 1988 114:53-7.

21. Asplund R, Aberg HE. Micturition habits of older people. Voiding frequency and urine volumes. Scand J Urol Nephrol 1992;26:345-9.

22. Lose G, Alling-Moller L, Jennum P. Nocturia in women. Am J Obstet Gynecol 2001;185:514-21.

23. Schatzl G, Temml C, Schmidbauer J, et al. Cross-sectional study of nocturia in both sexes: analysis of a voluntary health screening project. Urology 2000 56:71-5

24. Coyne KS, Zhou Z, Bhattacharyya SK, et al. The prevalence of nocturia and its effect on health-related quality of life and sleep in a community sample in the USA. BJU Int 2003;92:948-54.

25. Yoshimura K, Terada N, Matsui $\mathrm{Y}$, et al. Prevalence and risk factors for nocturia: analysis of a health screening program. Int J Urol 2004;11:282-7.

26. Van Dijk L, Kooij DG, Schellevis FG. Nocturia in the Dutch adult population. BJU Int 2002;90:644-8.

27. Rembratt A, Norgaard J, Andersson KE. Nocturia and associated morbidity in a community-dwelling elderly population. BJU Int 2003;92:726-30.

28. Bing MH, Moller LA, Jennum P, et al. Prevalence and bother of nocturia, and causes of sleep interruption in a Danish population of men and women aged60-8. years BJU Int 2006;98:599-604

29. Tikkinen KA, Tammela TL, Huhtala H, et al. Is nocturia equally common among men and women? A population based study in Finland. J Urol 2006a 175:596-600.

30. Mueller E, Latini J, Lux M, et al. Gender differences in 24-hour urinary diaries of asymptomatic North American adults. J Urol 2005;173:490-2.

31. Latini JM, Mueller E, Lux MM, et al. Voiding frequency in a sample of asymptomatic American men. J Urol 2004;172:980-4.

32. Hammarsten J, Hogstedt B. Hyperinsulinaemia as a risk factor for developing benign prostatic hyperplasia. Eur Urol 2001;39:151-8.

33. Asplund R, Aberg HE. Nocturia in relation to body mass index, smoking and some other life-style factors in women. Climacteric 2004;7:267-73.

34. Tikkinen KA, Auvinen A, Huhtala H, et al. Nocturia and obesity: a populationbased study in Finland. Am J Epideniol 2006b;163:1003-11.

35. Eckhardt MD, van Venrooij G, van Melick HM, et al. Prevalence and bothersomeness of lower urinary tract symptoms in benign prostatic hyperplasia and their impact on well-being. J Urol 2001;166:563-8.

36. Swithinbank LV, Abrams P. A detailed description, by age, of lower urinary tract symptoms in a group of communitydwelling women. BJU Int 2000;85: 19-24.

37. Chen FY, Dai YT, Liu CK, et al. Perception of nocturia and medical consulting behavior among community-dwelling women. Int Urogynecol J Pelvic Floor Dysfunct 2006; 10.1007/s00192-006-0167-x.

38. Fitzgerald MP, Mulligan M, Parthasarathy S. Nocturic frequency is related to severity of obstructive sleep apnea, improves with continuous positive airways treatment. Am J Obstet Gynecol 2006;194:1399-403.

39. Hunskaar S. Epidemiology of nocturia. BJU Int 2005;96:4-7. 